\title{
Automated Educative Nudges to Reduce Missed Assignments in College
}

\author{
Benjamin A. Motz ${ }^{1,2}$, Matthew G. Mallon ${ }^{3}$, Joshua D. Quick ${ }^{2,4}$ \\ ${ }^{1}$ Department of Psychological and Brain Sciences, Indiana University \\ ${ }^{2}$ eLearning Research and Practice Lab, Pervasive Technology Institute, Indiana University \\ ${ }^{3}$ University Information Technology Services, Indiana University \\ ${ }^{4}$ Learning Sciences Program, Department of Counseling and Educational Psychology, Indiana \\ University
}

\section{Author Note}

Benjamin Motz (iD 0000-0002-0379-2184

Joshua Quick (1D 0000-0001-5921-4984

Correspondence concerning this preprint should be addressed to Benjamin Motz.

Email:bmotz@indiana.edu 


\begin{abstract}
As institutions of higher education increasingly utilize online learning management systems, college students are asked to submit more assignments online. Under this regime, when most assignments are posted and submitted online, it is possible to know if a student is missing a submission for an imminent deadline, and to intervene proactively to reduce missed assignments and improve student outcomes. Toward this goal, we designed and evaluated a scalable targeted intervention: a mobile app that would deploy push notifications when students were missing submissions for assignments with imminent deadlines. Results from two experimental pilots demonstrate that this intervention system significantly decreased missed assignments compared with control notifications about instructor announcements to the class (in Experiment 1), and improved assignment adherence and course grades compared with courses that were not using the app (in Experiment 2). We discuss the benefits and theoretical implications of this behavioral guide rail, a purely informative proactive intervention to mitigate risk in advance of a negative outcome.
\end{abstract}

Keywords: Nudge, Reminder, Learning Management System, Mobile App, Prospective Memory 


\section{Automated Educative Nudges to Reduce Missed Assignments in College}

These days, our banks notify us when we have a low balance, our email clients suggest when we have forgotten to include attachments, and our cars alert us when we might be approaching a collision. While these systems do not entirely curtail our freedoms to overdraw our checking accounts or to crash into trees, they do mitigate risk from neglect or accidental misuse of the system. Can the same be said for educational technologies? In this article, we present an experimental study of this kind of proactive behavioral intervention in e-learning tools: a controlled evaluation of an automated system designed to help students avoid accidentally missing assignments.

Current proliferations of educational technologies, and in particular, web-based learning management systems (LMSs; e.g., Blackboard, Canvas, Bright Space), have created an abundance of opportunities for students to actively engage with their studies. In practice, many of these learning opportunities are implemented as graded assignments, and increasing utilization of LMSs [1] has resulted in a corresponding increase in the number of assignments that students are expected to complete. By practically all accounts, this increase is a good thing - more assignments means more active learning, more transparency about learning goals, and more opportunities for students to receive feedback (among others; [2, 3]). But the increasing institution of weekly assignments, practice quizzes, discussion forum posts, and problem sets also necessarily creates more material and deadlines for students to manage [4], and more opportunities for students to fall behind. In consequence, particularly in blended and online learning environments, a student's ability to stay on top of course material and requirements is a dominant factor in the student's retention in the course $[5,6]$. 
Traditional efforts to help students who are at risk of attrition usually start by identifying those whose past behaviors are substandard (e.g., [7]). After a student misses a few assignments, and misses the corresponding points toward their grade, conscientious instructors may reach out to support the student's future work, or classification models may levee risk flags targeting the student for remediation, under the mantle of improving student retention and success. But educational technology needn't stand idle until after deadlines are missed. If an e-learning system knows that an assignment deadline is upcoming, and a student has not submitted anything, proactive interventions might mitigate the possibility of risk before any risk is incurred. Instead of rescuing students only after lapses in engagement, learning technology might improve engagement before a problem is encountered.

\section{Intervention Science in Education}

A wide range of public policy domains, including environmental conservation, financial planning, and health and wellness, have been enriched by applying principles of psychological science and behavioral economics to reshape human behaviors toward more desirable outcomes [8]. Education, if one squints one's eyes, might be seen as preeminent in this frame, considering that the overarching goal of education -- to facilitate learning -- is, in effect, to facilitate behavior change. But while any lesson might be viewed as an educational "intervention," researchers have also been examining means of systemically improving educational outcomes, including those aimed at mitigating the long-range impacts of social problems on student success [9], assisting navigation through college and financial aid applications [10, 11], increasing attendance [12], improving students' self-regulation of learning [13, 14], and facilitating student motivation [15], among others [16]. But despite this range of efforts, there are no published examples of scalable interventions aimed at direct support of students' adherence to assigned learning activities. The 
relative scarcity of intervention todols for helping students stay on top of the college workload is particularly problematic, considering that contemporary e-learning environments provide more autonomy for students to complete (or to fail to complete) their coursework [17], and where completion of this coursework is a primary predictor of student engagement and success [18].

Behavioral science distinguishes two categories of interventions: nudges and boosts. Nudges are nonmonetary and nonregulatory interventions and policies aimed at changing behaviors, while still preserving freedom of choice in the target audience. Boosts share many similarities, but are instead aimed at empowering the target audience to change their own behaviors, with an emphasis on supporting internal processes and competencies [19]. If nudges and boosts were represented in a Venn diagram, the overlapping area would include interventions known as "educative nudges," such as reminders for upcoming appointments [20, 21].

As long as they refrain from explicitly instructing the student to do something, just-intime reminders about assignment deadlines will fall into this middle ground of "educative nudges," triggering behavior change by relying on the student's own capacity to act when shown pertinent information. In essence, the purpose of this information would be to help students remember to complete their schoolwork before an assignment deadline. A student who accidentally misses an assignment deadline has failed to remember a prospective memory (forgotten to perform an intended action) by a specific time (the target; [22]). Multiple cognitive processes are involved in remembering to perform an action, including periodic monitoring during the retention interval, as well as retrieval that is "spontaneously" triggered by attention to the target event [23]. 
Intuitively, we might assume that any reminder during the retention interval would reduce prospective memory failures, but not all reminders cause improvements. Even when a person is repeatedly informed about a prospective memory (rehearsal), they are not necessarily more likely to perform the action [24]. This may be because emphasis on the intended action alone does not reinforce the association between the action and the target event. In support of this view, substantial improvements are only seen when reminders also emphasize when the action should be performed [25]. Under these terms, we might hypothesize that simply informing students about course topics or pending assignments will be less effective than interventions that also highlight an imminent deadline. Additionally, not all students will need such assignment reminders. Brute force efforts to inform all students about all pending assignment deadlines will undoubtedly lead to desensitization and fatigue (e.g., [26]), if not outright annoyance. To avoid this issue, a reminder might be withheld until most students have already submitted the assignment (and thus could be excluded from the target audience), when it is sufficiently close to the assignment deadline that a student who hasn't submitted is likely to have forgotten to act.

\section{The Current Study}

The goal of the current study is to assess the benefit of proactive educative nudges for reducing missed assignments. Adherence to class assignments is, unsurprisingly, a principle predictor of student success and engagement in college $[17,18]$, and considering that assignments represent one of the most heavily utilized features of learning management systems [27], this is a fertile area for improving student success at scale.

In order to deliver interventions at a specific moment in time, we developed a smartphone app that was integrated with our institution's learning management system, Canvas (Instructure; Salt Lake City, UT). Eligible students could download the app, authenticate with their university 
credentials, and then receive push notifications about courses that were participating in the study. Sending interventions via push notifications within a smartphone app has many advantages, including that notifications are likely to be received immediately (unlike email, where the intervention may languish unread until a student decides to check their inbox), and that notifications can link directly to additional context within an app (unlike text messages, which have a limited range of user actions in response to the intervention). Moreover, mobile technology has been shown to be a particularly effective medium for supporting students in contemporary learning environments [28].

\section{Experiment 1}

The benefit of receiving a proactive assignment reminder on a mobile device is only meaningful in contrast to some comparison condition. In Experiment 1, we opted to compare automated assignment reminders with instructors' online announcements to the class. This is because, in the absence of any automated nudging intervention, instructors are responsible for sending their own reminders about upcoming assignments, and these announcements provide a business-as-usual control treatment with which to compare experimental automated reminders. This control condition was built within the same smartphone app: students received push notifications when an instructor posted an announcement to the class's Canvas site. We implemented the experimental contrast as a cross-over design, half the app's users (randomly assigned) received assignment reminders during the first half of the semester and then received push notifications informing them of instructor announcements in the second half of the semester, while the other half of the participants received the same treatments in the opposite 
order. Deidentified data and analysis scripts for this study are freely available at https://osf.io/qbt3y/.

Method

Participants. Seven undergraduate courses at four Indiana University campuses were selected to be eligible for this study during the Fall 2018 semester. These courses were chosen because they included at least weekly Canvas assignments that met the criteria for assignment reminders, and because they had enrollments greater than 35 students. Instructors of these courses announced the study in class or via email during the third week of the course, inviting students to download the reminder app with the incentive that participants would be entered into a drawing for one of 13 pairs of good-quality audio headphones. One course (with enrollment of 37 students) only had one student sign-up, and another course (with enrollment of 50 students) only had two students sign-up, so these two courses are removed from further analysis. The remaining 5 courses had cumulative enrollment of 950 students, and 133 of these students (14.0\%) downloaded the app and consented to participate. See Table 1 for additional details. All study procedures were approved by the Indiana University Institutional Review Board, and instructors did not know which students had registered for the study.

\section{TABLE I}

Courses involved in Experiment 1

\begin{tabular}{lcccc}
\hline \multicolumn{1}{c}{ Course Number: Topic } & Enrollment & Sign-ups & $\begin{array}{c}\text { Number of } \\
\text { Assignments }\end{array}$ & $\begin{array}{c}\text { Number of } \\
\text { Announcements }\end{array}$ \\
\hline K300: Statistical Techniques & 236 & $37(15.7 \%)$ & 16 & 14 \\
P211: Research Methods & 214 & $30(14.0 \%)$ & 9 & 7 \\
F205: Intro Forensic Science & 231 & $37(16.0 \%)$ & 32 & 22 \\
P103: General Psychology & 98 & $8(8.2 \%)$ & 20 & 17 \\
P101: General Psychology (Online) & 171 & $21(12.2 \%)$ & 111 & mean $=16.0$ \\
\hline Full Sample & 950 & $133(14.0 \%)$ & mean $=37.6$ \\
\hline \hline
\end{tabular}

Sign-ups are the number of enrolled students who downloaded the app and consented to participate. 
Procedure. In the third week of the semester, all enrolled students were invited (by their instructor) to join the study by downloading the free "IU Boost" app from either the Apple or Android app store. When the app first opened, students were prompted to log in with their official university credentials, to consent to participate in the research study, and to agree to share their student data with the researchers. Registered participants were then alternatingly assigned to either of two initial treatment groups: (1) receiving notifications when assignment deadlines were approaching with no submission; or (2) receiving notifications when the instructor posted a class announcement.

The current study employs a crossover design, such that halfway through the study, condition assignments flipped (students who initially received assignment reminders began receiving notifications about instructor announcements, and vice versa). This crossover design has two key benefits. First, it allows a more sensitive within-subject analysis of the difference between treatments for each participant while counterbalancing for order effects at the group level, and second, it has the ethical advantage of administering both treatments to all participants, preventing inequities between students due to condition assignment.

During the study, there were no deviations from the normal structure and content of the participating courses. The only manipulation was the type of notification that volunteer participants received within the app. The study concluded during the weekend prior to the last week of the academic term (12 weeks after the start of the study), which was one week before finals week.

Materials. The app, called "IU Boost" at the time of this study, was developed in the Expo framework (https://expo.io/), an open-source mobile development toolkit for React Native. See Figure 1 for screenshots. Every hour, a cloud function would submit requests to the Canvas 

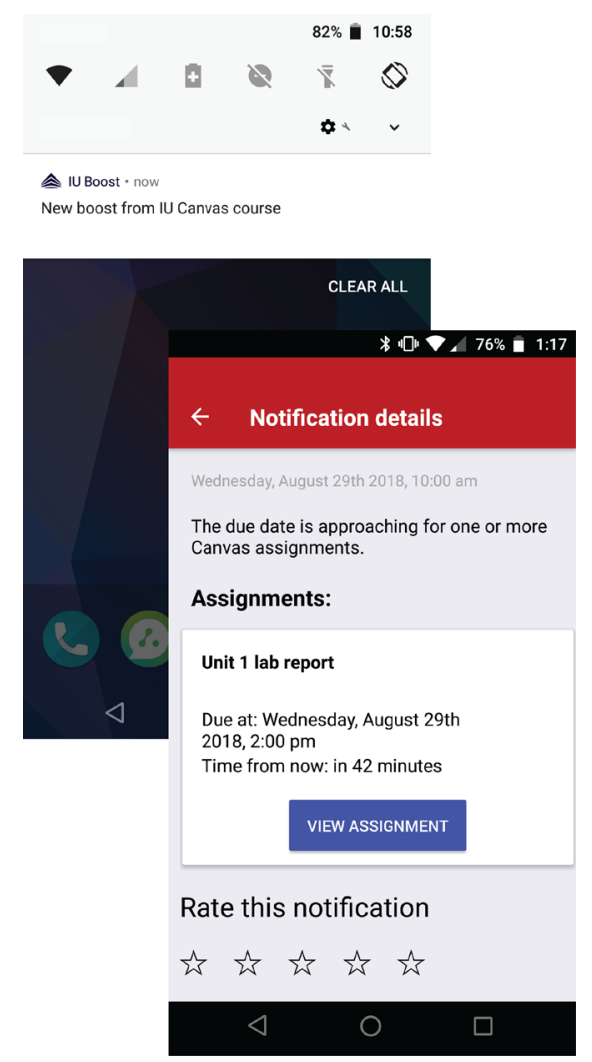

Fig. 1. App screenshots showing a notification for an assignment with an imminent deadline that is missing a submission.

API, retrieving details of registered users' upcoming assignments and instructor announcements in the pilot course sites. If the API revealed that an assignment deadline (for a graded individual assignment with online submission) had become due within the notification time window (4 hours) and lacked a submission, or if a new announcement was posted in a course site, the app deployed a push notification to the relevant users according to their assigned treatment. If a user was eligible to receive notifications about multiple assignments or announcements within the hour, the app grouped them into a single push notification.

For Experiment 1, all push notifications had the title "IU Boost" and the notification text was "New boost from IU Canvas course" (Figure 1). Tapping on the push notification opened the IU Boost app, which then showed notification details (participants could also navigate to the 
notification details by launching the app directly). Assignment reminders included the statement, "The due date is approaching for one or more Canvas assignments," followed by a card with additional assignment details (title, due date, time until due date, and a link to view the assignment) for each notified assignment. Instructor announcements included the statement, "A new announcement has been posted," followed by a card with the title of the announcement, and a link to view the announcement. Beneath the notification details, participants could rate the notification (a 5-point star rating); responding with a star rating also caused a textbox to appear where participants could type open-ended feedback.

Measured Variables. Students' activity related to Canvas assignments was passively recorded by default in Canvas, and these data were collected directly from our institution's Canvas data warehouse for study participants. For examining the effect of assignment reminders on student behavior, we focus on four separate dependent variables: (1) submission rate, the percent of assignments (only including those that were eligible to receive reminder notifications) for which the student provided a submission prior to the deadline; (2) percent score, the total points earned on these assignments, divided by the total points possible on these assignments; (3) views of assignment pages, the number of times that a student viewed the Canvas pages containing instructions for these assignments; and (4) submission time, the average amount of time, in hours, between a student's submission and the assignment's submission deadline.

Data Analysis. For each student and for each of the measured variables, we subtracted the average of observations when the student was assigned to receive instructor announcements from the average when a student was assigned to receive assignment reminders in the app. These raw difference scores represent the measured benefit of automated assignment reminders over instructor announcements for each individual participant, for each dependent variable. 
Difference scores were analyzed within the framework of a hierarchical Bayesian ANOVA. Bayesian estimation methods, such as those described herein, have different analytical goals than null hypothesis significance testing (for extended discussions, see [29, 30]). Rather than estimating the false positive rate under the idealized null model (as in a frequentist ANOVA), Bayesian estimation produces an informative posterior distribution of the most credible estimates of an experimental effect. A posterior distribution can be described by its highest density interval (HDI), which directly summarizes the tendency and uncertainty of a model parameter (analogous to but distinct from a confidence interval). In this particular hierarchical model, the benefit of assignment reminders is simultaneously estimated for each individual class, and also at the aggregate level for the total sample. For all parameters we use priors that are vague on the scale of the data (e.g., the priors for all experimental effects are wide distributions centered on 0.0), and thus the priors have very minimal effect on the posterior estimates. Full specification of the model parameters are available at https://osf.io/qbt3y/.

Participants' 5-point star ratings of notifications were also analyzed using Bayesian estimation, using the ordered-probit model described in [31], comparing two independent samples (ratings of assignment reminder notifications vs. ratings of instructor announcement notifications), since not enough ratings were provided for a within-subject comparison.

We used Markov chain Monte Carlo (MCMC) to sample the posterior distributions (60,000 steps across 4 chains, following 500 adaptation steps and 1,000 burn-in steps), using Gibbs sampling (JAGS; [32]), and the runjags package [33] for R. The effective sample size for the parameters of interest were no less than 20,000, well above the 10,000 recommended by [34]. Results

The study lasted 12 weeks. During this time, the instructors of the pilot courses assigned 

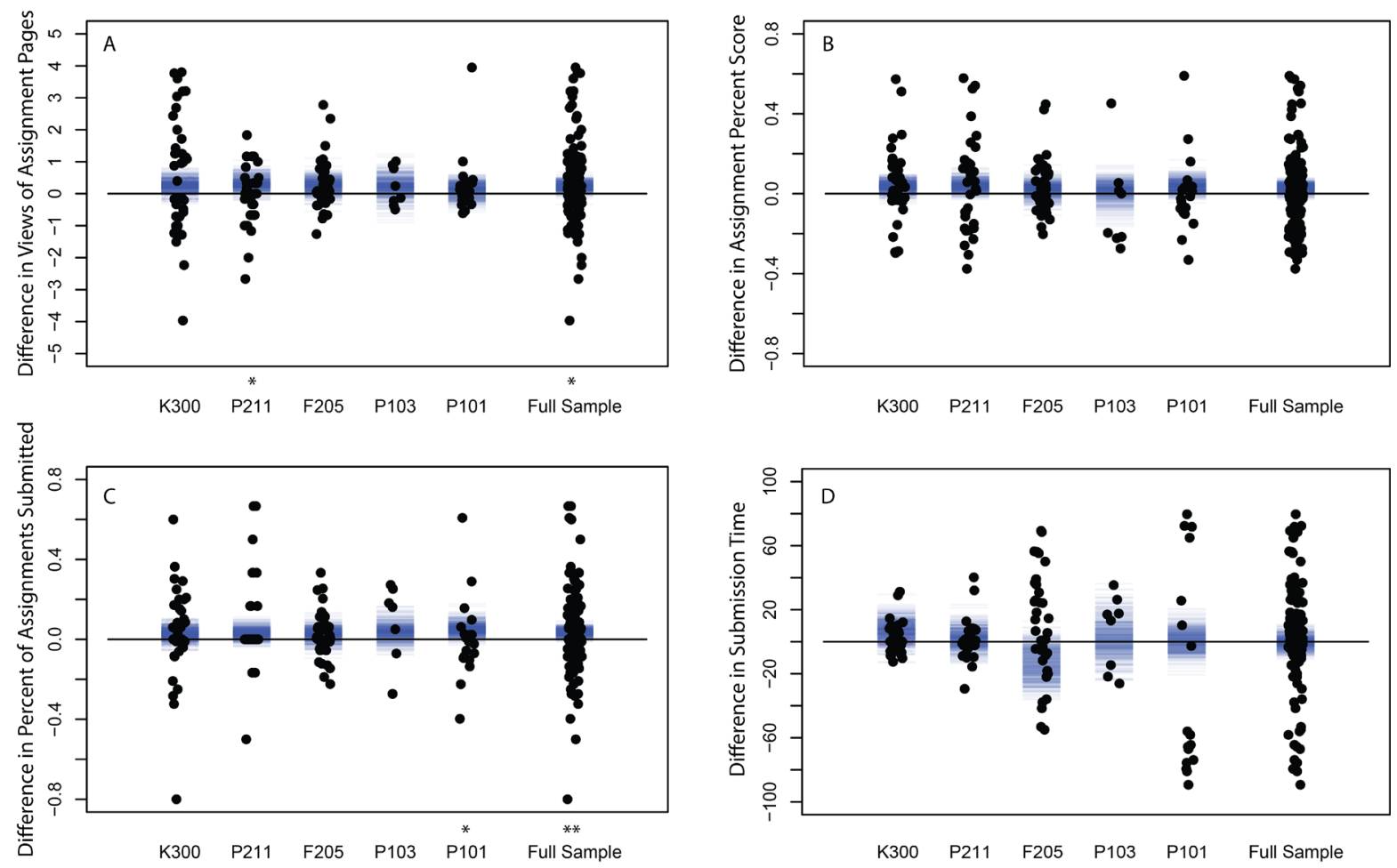

Fig 1. Effects of automated assignment reminders on student behavior and performance in Experiment 1. Each black dot represents a difference score for an individual student; difference scores are calculated by subtracting the average observation from when the student received assignment reminders from the average observation when the student received notifications about instructor announcements. Semi-transparent blue lines show credible model estimates of group means. $* 0 \notin 90 \% \mathrm{HDI} ; * * 0 \notin 95 \% \mathrm{HDI}$

an average of 37.6 assignments, and posted an average of 16.0 announcements (see Table 1). The exact number of notifications a participant received was affected by the course of enrollment, the time when a student first installed the app, and whether the student uninstalled the app at any time prior to the close of the study period.

When a participant was assigned to the control condition (to receive instructors' announcements), they received push notifications each time an announcement was posted to their course site. Across the full sample, the average number of announcement notifications deployed per participant was $5.0(S D=3.9)$. But when a student was assigned to the experimental condition to receive assignment reminders, they only received assignment reminders when there was no submission within 4 hours of the deadline. Thus, even though there were more 
assignments than announcements across all courses, the average number of assignment reminders deployed for each participant is lower than the number of announcement notifications, $4.2(S D=2.8)$.

Participants were invited to rate each notification on a 5-star rating scale, and participants submitted 49 such ratings during the study period. We analyzed these ratings separately for notifications about instructor announcements $(n=24)$ and assignment reminders $(n=25)$, using an ordered-probit model (in which ordered ratings are mapped onto a continuous distribution that can exceed the maximum rating value; [31]). The estimated mode of ratings about instructor announcements was 3.5 (95\% HDI: 3.0 to 4.1 ), and the mode of ratings about assignment reminders was 5.5 (95\% HDI: 3.2 to 9.1). Subtracting these estimates at every step in the MCMC chains produces a posterior distribution of the difference between these types of notifications. The mode of these difference estimates is 1.9 , but this effect was modest in proportion to uncertainty (considering the relatively small number of ratings received in each condition), the 95\% HDI (-0.4 to 5.6) included 0, however, the 90\% HDI (0.005 to 4.9) excluded 0 , indicating a trend for participants to provide higher ratings to assignment reminders.

The actual behaviors of the participants provided a clearer picture of the benefit of automated assignment reminders (see Figure 2). Across all courses, the most noteworthy finding was that participants had a significant tendency to submit more assignments $(3.7 \%$ increase; $95 \%$ HDI: 0.01 to $7.3 \%$ ) when they received assignment reminders than when they received instructor announcements. When they received these automated assignment reminders, participants also had a slight trend to view assignments more frequently (0.23 more views; 90\% HDI: 0.02 to 0.43). In each of the pilot courses, students received directionally-higher assignment scores when students received assignment reminders (overall difference mode $=3.1 \%$ improvement), but 
variability caused these difference estimates in assignment scores to overlap zero (90\% HDI: -0.1 to $6.0 \%$ ). There were no differences in the time when students submitted assignments between treatment conditions (difference mode $=0.06$ hours; $90 \%$ HDI: -6.4 to 5.7 ).

\section{Discussion}

When students received automated assignment reminders on their smartphones, they were more likely to submit their assignments than when they received notifications about instructor announcements. This is noteworthy, considering that instructors have privileged insights into the standards of their courses, and that students also tended to receive more notifications regarding instructor announcements, but these were less effective than automated reminders at increasing views of assignment pages and submissions of assignments. The current results support and extend previous research on prospective memory, demonstrating that periodic announcements about coursework may be less effective than automated educative nudges that highlight imminent targets when students need to complete their coursework.

However, even while instructor announcements may be less effective than targeted assignment reminders for improving assignment submission rates, these are not mutuallyexclusive in practice. There is no reason that an automated intervention system should only deploy one or the other. Rather, there may be additive benefits to providing both kinds of educative nudges (reminders about upcoming assignment deadlines, and notifications for new announcements from the instructor) within a mobile notification tool. A limitation of Experiment 1 was that it could not estimate the cumulative benefit of these proactive notifications compared with courses where the app is not enabled. To inform a practical estimate of the benefits of this kind of automated system, it would be useful to measure cases where the automated system is active, contrasted with students' behaviors in comparable courses where the tool is not active. 
Such a contrast shares similarity with a clinical research model known as "pragmatic trials" [35], where the goal is to estimate the general effectiveness of a broad intervention in natural practice, not to explain how an intervention confers benefits in a limited context. Another limitation of Experiment 1 was its sample size; too few students signed-up for the pilot to draw strong inferences about the benefits of assignment reminders on course performance. The goal of Experiment 2 is to address these limitations, with a quasi-experimental analysis of the aggregate practical benefits of a system for deploying these kinds of automated notifications, at a much larger scale.

\section{Experiment 2 (Quasi-Experiment)}

Buoyed by directionally-positive results from Experiment 1, our institution decided to make the smartphone app available to any instructor who wanted the app to be activated in their courses. This open pilot made possible a much larger sample size than in Experiment 1, but considering that participation was predicated on students receiving the potential benefits of the app, we decided not to implement a randomized controlled trial research design. We felt it was inappropriate to withhold assignment reminders for any app users in this broader release, which would have stunted the benefits of the app for the students and the instructors who volunteered. For this reason, Experiment 2 uses a quasi-experimental design. For each student who uses the app, we contrast their behaviors in classes where the app is operational (i.e., where the instructor has opted-in for the app to be available) against their behaviors in classes where the app is not active (i.e., where the instructor could have opted-in, but did not). This analysis uses regression adjustment to control for structural differences (besides the availability of the app) between these two different kinds of courses. 


\section{Method}

Participants. Instructors of 1,632 courses, with diverse representation from all Indiana University campuses, signed-up to make the app available to their students during the Spring 2019 semester. All students enrolled in these courses received up to three email invitations to download the app and to consent to participate in research on the app's benefits (no additional incentives; invitations were withheld from students who had already activated the app). Every third student who activated the app was sequentially assigned to a baseline treatment condition, which would only receive assignment reminders and announcement notifications as with Experiment 1 (new features introduced in the app during Spring 2019 semester were withheld from these students). Students were subsequently excluded from analysis if they withdrew from the course where the app was enabled, or if they were not enrolled in at least one other course where the app was not enabled. There were 564 students who met the inclusion criteria and who were assigned to this baseline treatment, enrolled in 420 courses where the app was enabled. These students were also enrolled in 1,289 other courses, where the app would have been eligible to be enabled, but the instructor had not opted-in. Overall, the average student in this baseline treatment was enrolled in 1.48 courses where the app was enabled, and in 3.04 courses where the app was not enabled. Students downloaded and activated the app 14 days after the start of the semester on average. All study procedures were approved by the Indiana University Institutional Review Board, and instructors did not know which students were using the app.

Procedure. All Indiana University instructors-of-record, for any credit-bearing course using Canvas, were eligible to opt-in for the app to be available to their students during the Spring 2019 semester, using a simple online sign-up form. Instructors were invited to opt-in through teaching center email newsletters, learning technology listservs, and a global 
TABLE 2

Courses involved in Experiment 2

\begin{tabular}{|c|c|c|c|c|c|}
\hline Class Type & Number of Classes & Avg Enrollment & $\begin{array}{c}\text { Avg Number of } \\
\text { Assignments }\end{array}$ & $\begin{array}{l}\text { Avg Number of } \\
\text { Announcements }\end{array}$ & $\begin{array}{l}\text { Avg Duration } \\
\text { Between } \\
\text { Submission and } \\
\text { Deadline (hours) }\end{array}$ \\
\hline App Enabled & 420 & 52.0 & 19.7 & 16.1 & 58.9 \\
\hline App Not Enabled & 1289 & 52.7 & 16.9 & 13.2 & 47.9 \\
\hline Full Sample & 1861 & 52.5 & 17.6 & 13.9 & 53.3 \\
\hline
\end{tabular}

The 564 students who were using the app during the Spring 2019 semester were enrolled in 420 different courses where the app was enabled, and 1289 courses where the app was not enabled. Classes where the app was enabled were those where the instructor opted-in for the app to be available. Classes where the app was not enabled were those courses that were eligible for the app to be enabled, but the instructor had not opted-in. Values shown are controlled as fixed effects in analytical model.

announcement that appeared on all instructors' Canvas dashboards at the start of the semester.

When an instructor opted-in, all the instructor's courses (where the instructor was assigned the "Teacher" role in Canvas) were enabled within the app, and the students enrolled in these courses received a series of up to three emails (one per week) inviting them to download and activate the app.

There was no manipulation of treatment in Experiment 2. All 564 students received access to the same functionality within the app from the time when they activated the app through the end of the Spring 2019 semester.

For this quasi-experiment, we contrast students' behaviors and performance in courses where the app had been enabled, with the same students' behaviors in courses where the app had not been enabled, but would have been eligible (the course used Canvas and the course was credit-bearing). In our analysis, we control for differences between these courses that are unrelated to the availability of the app (using regression adjustment, see Data Analysis, below), such as the number of assignments, the number of instructor announcements, the average time prior to the deadline when assignments were submitted, and the average class enrollment. See Table 2 for comparisons between these values. 
Materials. Similarly to Experiment 1, students downloaded the app "IU Boost" from the app stores free of charge. There were some modest differences from Experiment 1 in the app's behavior, primarily intended to support the app's utilization across a wider range of courses:

- The app was customizable. Students could turn different types of notifications on or off (e.g., notifications about instructor announcements could be disabled for a single course), or they could mute all notifications for entire courses. Students could also, on a courseby-course basis, set how long before a deadline a due date reminder should be deployed (default was 4 hours, customizable to 1-24 hours).

- There were three different types of available notifications to the students in this baseline treatment group, and all were enabled by default throughout the semester. Unlike Experiment 1, (where the text of every push notification was "New boost from IU Canvas course"), the push notification included variable text according to the notification type: (1) Due date reminders, as described in Experiment 1, where the notification text was "The due date is approaching for one or more Canvas assignments." (2) Instructor announcement notifications, as described in Experiment 1, where the notification text was "A new announcement has been posted." And (3) a Daily assignment digest, deployed at 1:00pm local time, which contained a list of all assignments due within the next 24 hours. The text of the notification was " $[\mathrm{N}]$ Canvas assignment[s] [is/are] due in the next 24 hours in your enabled courses. All due assignments, even those already submitted, are included." -- where [N] was the number of assignments due. This daily digest notification was created to provide reminders for assignments where Canvas would lack submission information (e.g., assignments carried out in a textbook publisher's separate learning platform). 
- To improve the timeliness of notifications, the app queried the Canvas API and deployed notifications every 30 minutes, rather than every 60 minutes.

Measured Variables. As in Experiment 1, passively-recorded data were collected directly from our institution's Canvas data warehouse for students who consented to participate when they activated the app. For Experiment 2, we focus on the following two dependent variables: (1) submission rate, the percent of assignments (only including those that were eligible to receive reminder notifications) for which the student provided a submission prior to the deadline; and (2) final score, the estimate of the student's final percent score in the course, according to the points, assignment weights, and grading calculations defined by the instructor in Canvas. We omitted the more granular measures used in Experiment 1 (views of assignment pages, submission time, and assignment score), due to the substantially larger and more diverse sample in Experiment 2. For example, the sample in Experiment 2 includes a large number of discussion assignments (where views involve both reading the assignment and also reading others' posts, even after one's submission), external tool assignments (where Canvas may not have accurate logs of whether students view the assignments), assignments without pressing open/close dates (where submissions occur weeks before the deadline), pass/fail assignments (where the assignment score is binary), letter grade assignments (where the assignment score is ordinal), and so on.

Data Analysis. For each student, we calculated the two measured variables, submission rate and final score, as means in two separate bins: for courses where the app was enabled, and where the app was not enabled. 

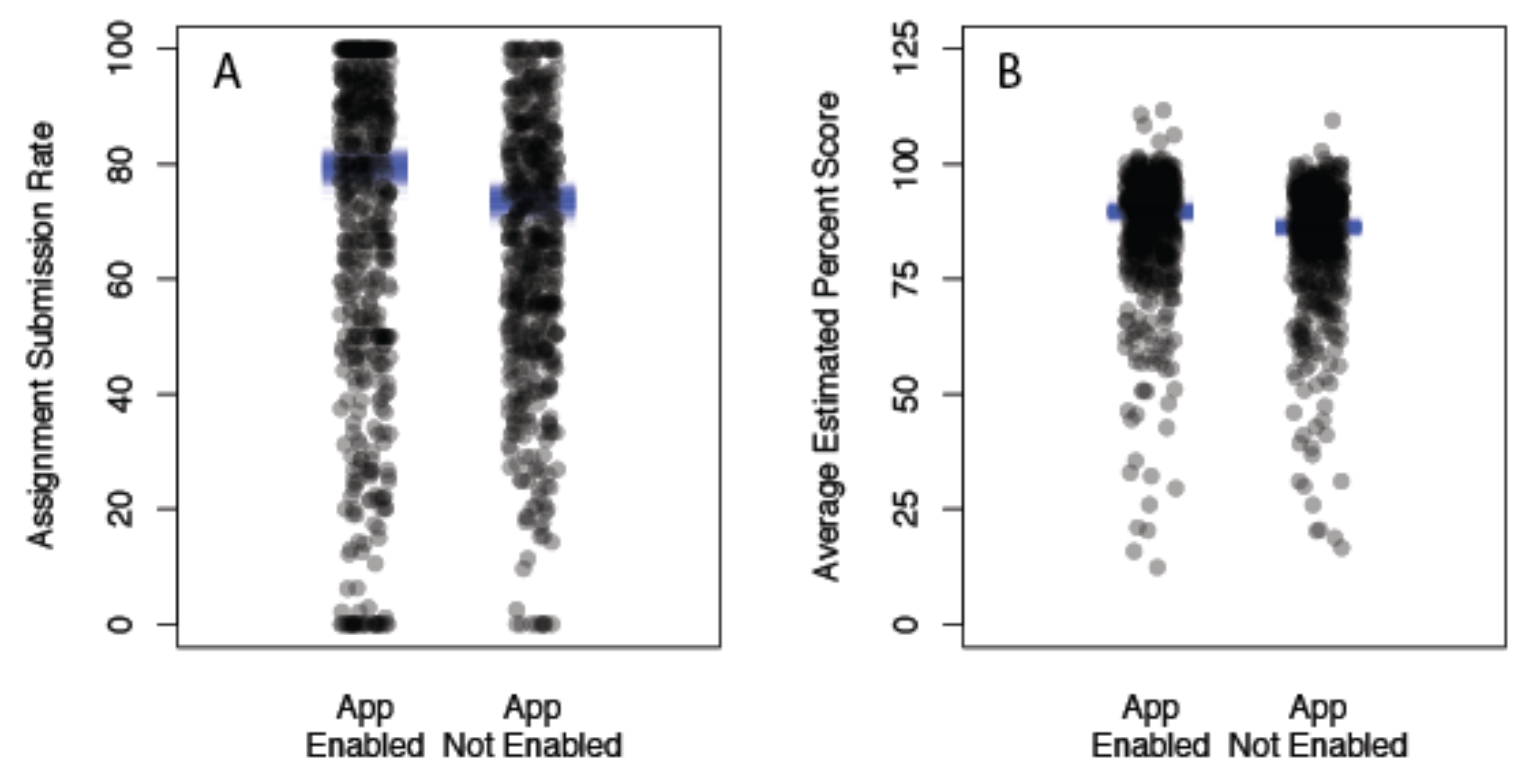

Fig 3. Effects of automated assignment reminders on student behavior and performance in Experiment 2. Dots represent observations for individual student enrollments, for (A) percent of assignments submitted, and (B) estimated cumulative percent score. Semi-transparent blue lines show credible model estimates of group means after controlling for covariates. In both plots, the differences between groups are significant.

These measures were analyzed within the framework of Bayesian generalized linear mixed model, using the brms package for R [36]. Similar to the analysis described in Experiment 1 , this method also produces an informative posterior distribution of the most credible estimates of an experimental effect. But in this particular framework, the effect of the app (enabled vs. notenabled) on a measured variable (e.g., a student's submission rate) is estimated as a coefficient in a general linear mixed model, with additional coefficients for regression adjustment (parameters accounting for variance due to the average number of assignments, the number of instructor announcements, the average time prior to the deadline when assignments were submitted, and the average class enrollment; see Table 2), with a random-effect term for the individual student, and with observations weighted by the number of classes that comprised these averages. The priors of all regression parameters were wide normal distributions with mean zero, and standard 
deviation 5-times the observed standard deviation of the dependent variable for the full sample. Full specification of the model parameters is available at https://osf.io/qbt3y/.

As is the default in brms, model parameters were estimated using the No-U-Turn Sampler (NUTS; [37]), using 4,000 steps across 4 chains, following 2,000 warm-up iterations in each chain. Model convergence was evaluated by visual inspection of the chains, and by the potential scale reduction factor, $\hat{R}$ [38]. $\hat{R}$ was less than 1.01 for each regression coefficient (values of 1.00 reflect ideal model convergence).

Results

Experiment 2 lasted for the full span of the 16-week spring 2019 academic semester. During this time, participants who met the Experiment 2 inclusion criteria had an average of 77.7 graded assignments with deadlines on Canvas, which is roughly typical of our institution's overall average.

Of the 564 students who activated the app, 510 received some form of notification during the study period (the additional 54 participants may have deleted or muted the app, but these participants are still included in the study sample). In total, 17,903 notifications were deployed to these students, corresponding to an average of 35.1 notifications per notified participant. Of these, $15.3 \%$ were reminders about imminent deadlines, $55.0 \%$ were announcements, and 29.7\% were digests of assignments due within the next 24 hours. Thus, during the study period, the average student received about 5 push notifications about assignments with imminent deadlines that were missing a submission, roughly comparable to the deadline reminder "dosage" in Experiment 1.

For all participants' course enrollments during the spring 2019 semester, we identified the percent of graded assignments with a deadline that had a submission record. In this broad 
sample of 1,861 courses, a range of assignment types would be expected, including extra-credit assignments and assignments that merely constituted participation points, as well as final papers, for example. The intercept of the linear model analyzing submission rates for these assignments was estimated to be $73.7 \%$ (95\% HDI: 70.8 to $76.5 \%$ ). With controls for structural properties of the different courses (listed in Table 2), the coefficient corresponding to the effect of the app was a 5.7\% increase in assignment submission rate (95\% HDI: 4.3 to 7.1 ; see Figure $3 \mathrm{~A})$. In this model, the parameter controlling for the number of assignments in the course was also significant, a $0.32 \%$ decrease (95\% HDI: -0.40 to -0.24 ) in assignment submission rate for every additional assignment in a course. In other words, the more assignments in a course, the lower the percent of assignments submitted in the course. Full model fits are available at

\section{https://osf.io/qbt3y/.}

Participants' final cumulative percent scores, from each course enrollment during the spring 2019 semester, were collected from Canvas, according to the point values and weighting schemes implemented by the instructor. Although these values are not the official grades recorded on the students' transcripts, these percent scores nevertheless provide a fair measure of participants' course performance, with more granularity than would be possible with coarse, ordinal letter grades. The intercept of the cumulative percent score linear model was estimated to be $86.3 \%$ (95\% HDI: 84.8 to $87.8 \%$ ). Again, with controls for structural differences between courses, the coefficient corresponding to the effect of the app was a $3.36 \%$ improvement $(95 \%$ HDI: 2.64 to $4.09 \%$; see Figure 3B) in course performance in courses where the app was enabled. This effect size is remarkably close to the $3.1 \%$ improvement in assignment scores observed in Experiment 1, although in Experiment 2 there is much less uncertainty in this effect estimate, owing to the larger sample size. 


\section{Discussion}

Experiment 2 investigated the cumulative benefit of automated notifications about course content at a much larger scale than Experiment 1 . The analysis revealed that students reliably submitted assignments more often, and received reliably higher grades, in courses where automated notifications were enabled, compared with courses where these notifications were not enabled. Regression adjustments for differences between these courses reduces the possibility that this effect was caused by course-level covariates such as enrollment size, number of assignments, number of announcements, or the timing of assignment submissions.

In contrast to Experiment 1, which estimated the benefit of automated deadline reminders over instructor announcements, Experiment 2 estimated the cumulative benefit of both types of notifications, comingled in the same pragmatic treatment. Considering that instructor announcements do have benefits for student motivation (e.g., [39]) and behavior (e.g., [40]), it makes sense that the estimated improvement in assignment submission rates (compared with no notification whatsoever) in Experiment $2(+5.7 \%)$ was higher than Experiment $1(+3.7 \%)$. Alternatively, considering the observation that courses with more assignments have lower assignment submission rates, the "stakes" for individual assignment submissions in Experiment 1 (where courses had a relatively large number of assignments) were lower than the broader sample in Experiment 2, which may have attenuated the benefit of deadline reminders in Experiment 1. Regardless, results from both experiments suggest reliable benefits from these automated notifications.

\section{General Discussion}

Across two experiments, we observed significant improvements in assignment 
submission rates when students received automated proactive nudges about their schoolwork. Experiment 1 was a within-subject randomized controlled trial, where it was observed that educative nudges about proximal deadlines significantly improved assignment submission rates over control notifications (instructor announcements). Experiment 2 used a quasi-experimental design at a larger scale, and observed significant improvements in assignment submission rates and grades in courses where the mobile notification system was enabled. In total, these results provide strong evidence of the efficacy of automated nudges for improving adherence to assigned coursework.

Students are expressly responsible for staying on top of their schoolwork. But with an increasing volume of assignments in blended and online learning environments, the difficulties of managing the workload is a leading cause of attrition [5]. While periodic notifications about announcements from an instructor may be of some benefit, the results of Experiment 1 suggest that automated proactive nudges about upcoming assignment deadlines are significantly more efficacious for improving assignment submission rates — even while instructor announcements are more frequent. By delivering a nudge that is proximal to an upcoming deadline, the automated notification system has the advantage of highlighting the time when action is required. In this regard, the current results support theory from prospective memory research, stating that reminders will be most effective not when they are repeated, but when they highlight the eliciting target event [25].

Automated interventions to support student engagement can take many forms. The current study evaluated the benefit of a mobile reminder system, designed to be maximally portable across any course containing an assignment with a due date. Alternative systems, such as OnTask [41], allow the instructor to trigger custom support messages specific to the learning 
context within the course. These more contextual tailored interventions may provide added benefit (e.g., as observed in health care, [42]), but they rely on individual instructors to set them up, presenting a bottleneck to their broad utilization. We see value in both approaches, providing tools for instructors to support student learning within the idiosyncrasies of specific courses, while also designing general-purpose interventions that provide behavioral guiderails at scale. Automated interventions can also operate at different time horizons. While the current study aimed to reduce missed assignments in the hours immediately following the intervention, other approaches aim to improve distal outcomes, such as major choice, end-of-semester grades, persistence, and interest $[9,43]$. Some advocate that these longer-range interventions aimed at changing mindset or forming habits (rather than affecting behaviors in the immediate vicinity of the intervention) represent a preferred target for nudging [44]. Future studies might compare whether these longer-range interventions complement or enhance the benefits of short-term reminders.

The contents of behavioral interventions commonly face issues related to paternalism. Paternalism is a shifting concept in behavioral science [45], but generally refers to a belief that individuals will tend to make inferior choices, and that these choices could be improved with the assistance of a better-informed agent. Interventions that steer behaviors toward what the agent believes is "good" for an individual are more paternalistic, while interventions that facilitate an individual's cognitive capacity to make an informed decision are less paternalistic. Heavyhanded paternalistic approaches can present ethical challenges, such as when a government or a market institution claims to know what's best for its constituents [46] (c.f. [47]). But in Western educational systems, where students are expected to seek the instruction of an informed faculty, the faults of paternalism are less clear. For example, even while administrators advocate against 
views of students as deficient (e.g., [48]), empirical research demonstrates that some paternalistic policies are effective at closing achievement gaps and increasing college preparation in inner-city schools $[49,50]$. Similarly, an instructor's tailored suggestion of what a student should do can be more effective at improving student engagement than purely informative interventions [40], and instituting a firm schedule of assignment deadlines leads to improved performance over flexible student-selected assignment deadlines [51]. These successful paternalistic approaches share common features of being highly personalized and contextual, and thus difficult to implement at scale. Less paternalistic approaches, such as growth mindset interventions (e.g., [52]) may be more appropriate for facilitating student engagement and achievement across diverse courses and institutions. In the same vein, we designed the automated, general-purpose intervention in the current study to be strictly educative, with no instruction or suggestion for how the student should behave, only a reminder statement that "the due date is approaching."

This app therefore presents a "middle-ground" approach between heavy paternalistic and non-paternalistic approaches. The capacity to grant student agency enables flexible implementation of the tool for students and instructors to make informed decisions based on contextual information that more paternalistic approaches tend to ignore. Further, the structure of the tool enables guiding practices for how the tool is to be used in an educative manner.

The educative nudge described in this study might be framed as a behavioral guide rail, a purely informative proactive intervention to mitigate risk in advance of a negative outcome. The positive results warrant optimism that smartphone apps represent a promising platform for deploying these kinds of timely, automated, data-driven interventions at scale. 


\section{Acknowledgment}

We would like to thank Matthew Gunkel, Tori Scott, Rick Hullinger, Gina Londino-Smolar, Dennis Rodriguez, Maggie Ricci, Erik Scull, Chris Foley, John Gosney, and Stacy Morrone, for their insights, assistance, and support of this project. 


\section{References}

[1] J. Pomerantz and D. C. Brooks, "ECAR Study of Faculty and Information Technology," ECAR, Louisville, CO, USA, 2017.

[2] U. Trautwein, "The homework-achievement relation reconsidered: Differentiating homework time, homework frequency, and homework effort," Learning and Instruction, vol. 17, no. 3, pp. 372-388, 2007.

[3] J. Gikandi, D. Morrow and N. Davis, "Online formative assessment in higher education: A review of the literature," Computers in Education, vol. 57, no. 4, pp. 2333-2351, 2011.

[4] J. Sweller, J. van Merrienboer and F. Paas, "Cognitive architecture and instructional design," Educational Psychology Review, vol. 10, no. 3, pp. 251-296, 1998.

[5] P. Bawa, "Retention in online courses: Exploring issues and solutions-A literature review," Sage Open, vol. 6, no. 1, pp. 1-11, 2016.

[6] M. Herbert, "Staying the course: A study in online student satisfaction and retention," Online Journal of Distance Learning Administration, vol. 9, no. 4, 2006.

[7] R. Barber and M. Sharkey, "Course correction: Using analytics to predict course success," in Proceedings of the 2nd International Conference on Learning Analytics and Knowledge (LAK'12), New York, NY, USA, 2012.

[8] S. Benartzi, J. Beshears, K. L. Milkman, C. R. Sunstein, R. H. Thaler, M. Shankar, W. Tucker-Ray, W. J. Congdon and S. Galing, "Should governments invest more in nudging?," Psychological Science, vol. 28, no. 8, pp. 1041-1055, 2017. 
[9] G. Walton, "The new science of wise psychological interventions," Current Directions in Psychological Science, vol. 23, no. 1, pp. 73-82, 2014.

[10] B. L. Castleman and L. C. Page, "Summer Nudging: Can Personalized Text Messages and Peer Mentor Outreach Increase College Going Among Low-Income High School Graduates?," Ed Policy Works Working Paper No. 9, Charlotesville, VA, 2013.

[11] K. A. Bird, B. L. Castleman, J. Goodman and C. Lamberton, "Nudging at a national scale: Experimental evidence from a FAFSA completion campaign," Ed Policy Works Working Paper No. 54, Charlottesville, VA, 2017.

[12] T. Rogers and A. Feller, "Reducing student absences at scale by targeting parents' misbeliefs," Nature Human Behavior, vol. 2, pp. 335-342, 2018.

[13] J. L. Nietfeld, L. Cao and J. W. Osborne, "The effect of distributed monitoring exercises and feedback on performance, monitoring accuracy, and self-efficacy," Metacognition and Learning, vol. 1, no. 2, p. 159, 2006.

[14] G. D. Saenz, L. Geraci and R. Tirso, "Improving metacognition: A comparison of interventions," Applied Cognitive Psychology, vol. 33, no. 5, pp. 918-929, 2019.

[15] R. A. Lazowski and C. S. Hulleman, "Motivation interventions in education: A metaanalytic review," Review of Educational Research, vol. 86, no. 2, pp. 602-640, 2016.

[16] A. S. Bryk, L. M. Gomez, A. Grunow and P. G. LeMahieu, Learning to improve: How America's schools can get better at getting better, Cambridge, MA: Harvard Education Press, 2015.

[17] H. Kauffman, "A review of predictive factors of student success in and satisfaction with online learning," Research in Learning Technology, vol. 23, pp. 1-13, 2015. 
[18] B. A. Motz, J. Quick, N. Schroeder, J. Zook and M. Gunkel, "The validity and utility of activity logs as a measure of student engagement," in Proceedings of the 9th International Conference on Learning Analytics \& Knowledge (LAK19), New York, NY, USA, 2019.

[19] T. Grüne-Yanoff and R. Hertwig, "Nudge versus boost: How coherent are policy and theory?," Minds and Machines, vol. 26, no. 1, pp. 149-183, 2016.

[20] C. Sunstein, Why nudge? The politics of libertarian paternalism, New Haven, CT: Yale University Press, 2014.

[21] R. Hertwig and T. Grüne-Yanoff, "Nudging and boosting: Steering or empowering good decisions," Perspectives on Psychological Science, vol. 12, no. 6, pp. 973-986, 2017.

[22] M. McDaniel and G. Einstein, "Strategic and automatic processes in prospective memory retrieval: a multiprocess framework," Applied Cognitive Psychology, vol. 14, no. 7, pp. S127-S144, 2000.

[23] G. Einstein and M. McDaniel, "Prospective memory: Multiple retrieval processes," Current Directions in Psychological Science, vol. 14, no. 6, pp. 286-290, 2005.

[24] O. Vortac, M. Edwards and C. Manning, "Functions of external cues in prospective memory," Memory, vol. 3, no. 2, pp. 201-219, 1995.

[25] M. Guynn, M. McDaniel and G. Einstein, "Prospective memory: When reminders fail," Memory \& Cognition, vol. 26, no. 2, pp. 287-298, 1998.

[26] J. Cash, "Alert fatigue," American Journal of Health-System Pharmacy, vol. 66, no. 23, pp. 2098-2101, 2009. 
[27] S. Lonn and S. D. Teasley, "Saving time or innovating practice: Investigating perceptions and uses of Learning Management Systems," Computers \& Education, vol. 53, no. 3, pp. 686-694, 2009.

[28] Y. T. Sung, K. E. Chang and T. C. Liu, "The effects of integrating mobile devices with teaching and learning on students' learning performance: A meta-analysis and research synthesis," Computers \& Education, vol. 94, pp. 252-275, 2016.

[29] J. Kruschke and T. Liddell, "Bayesian data analysis for newcomers," Psychonomic Bulletin \& Review, vol. 25, no. 1, pp. 155-177, 2018.

[30] J. Kruschke and T. Liddell, "The Bayesian New Statistics: Hypothesis testing, estimation, meta-analysis, and power analysis from a Bayesian perspective," Psychonomic Bulletin \& Review, vol. 25, no. 1, pp. 178-206, 2018.

[31] T. Liddell and J. Kruschke, "Analyzing ordinal data with metric models: What could possibly go wrong?," Journal of Experimental Social Psychology, vol. 79, pp. 328-348, 2018.

[32] M. Plummer, "JAGS: A program for analysis of Bayesian graphical models using Gibbs sampling," in Proceedings of the 3rd International Workshop on Distributed Statistical Computing, 2003.

[33] M. Denwood, "runjags: An R Package providing interface utilities, model templates, parallel computing methods and additional distributions for MCMC models in JAGS," Journal of Statistical Software, vol. 71, pp. 1-25, 2016.

[34] J. Kruschke, Doing Bayesian data analysis: A tutorial with R, JAGS, and Stan (2nd Ed.), Cambridge, MA, USA: Academic Press, 2014. 
[35] N. Patsopoulos, "A pragmatic view on pragmatic trials," Dialogues in Clinical Neuroscience, vol. 13, no. 2, pp. 217-224, 2011.

[36] P. Bürkner, "brms: An R Package for Bayesian Multilevel Models Using Stan," Journal of Statistical Software, vol. 80, no. 1, pp. 1-28, 2017.

[37] M. Hoffman and A. Gelman, "The No-U-Turn Sampler: Adaptively setting path lengths in Hamiltonian Monte Carlo," The Journal of Machine Learning Research, vol. 15, no. 1, pp. 1593-1623, 2014.

[38] A. Gelman and D. Rubin, "Inference from Iterative Simulation Using Multiple Sequences," Statistical Science, vol. 7, no. 4, pp. 457-472, 1992.

[39] A. Legg and J. Wilson, "E-mail from professor enhances student motivation and attitudes," Teaching of Psychology, vol. 36, no. 3, pp. 205-211, 2009.

[40] J. Eyink, B. Motz, G. Heltzel and T. Liddell, "Self-regulated studying behavior, and the social norms that influence it," Journal of Applied Social Psychology, 2019.

[41] A. Pardo, K. Bartimote, S. B. Shum, S. Dawson, J. Gao, D. Gaševic, S. Leichtweis, D. Liu, R. Martinez-Maldonado, N. Mirriahi, A. Moskal, J. Schulte, G. Siemens and L. Vigentini, "OnTask: Delivering data-informed, personalized learning support actions," Journal of Learning Analytics, vol. 5, no. 3, pp. 235-249, 2018.

[42] F. Muench and A. Baumel, "More than a text message: Dismantling digital triggers to curate behavior change in patient-centered health interventions," Journal of Medical Internet Research, vol. 19, no. 5, p. e147, 2017. 
[43] J. Harackiewicz and S. Priniski, "Improving student outcomes in higher education: The science of targeted intervention," Annual Review of Psychology, vol. 69, no. 1, pp. 409$435,2018$.

[44] K. Stawarz, A. Cox and A. Blandford, "Beyond self-tracking and reminders: Designing smartphone apps that support habit formation," in Proceedings of the 33rd Annual ACM Conference on Human Factors in Computing Systems (CHI '15), New York, NY, USA, 2015.

[45] D. Hausman, "Efficacious and ethical public paternalism," Review of Behavioral Economics, vol. 5, no. 3-4, pp. 261-280, 2018.

[46] M. Rizzo and D. Whitman, "Little brother is watching you: New paternalism on the slippery slopes," Arizona Law Review, vol. 51, no. 3, pp. 685-739, 2009.

[47] S. Conly, Against Autonomy: Justifying Coercive Paternalism, Cambridge: Cambridge University Press, 2012.

[48] B. White, "Beyond a Deficit View," Inside Higher Ed, 19 April 2016.

[49] D. Whitman, Sweating the Small Stuff: Inner-City Schools and the New Paternalism, Washington, DC, USA: Thomas B Fordham Institute Press, 2008.

[50] K. McDermott and K. Nygreen, "Educational new paternalism: Human capital, cultural capital, and the politics of equal opportunity," Peabody Journal of Education, vol. 88, no. 1, pp. 84-97, 2013.

[51] D. Ariely and K. Wertenbroch, "Procrastination, deadlines, and performance: Self-control by precommitment," Psychological Science, vol. 13, no. 3, pp. 219-224, 2002. 
[52] D. Yeager, P. Hanselman, G. Walton, J. Murray, R. Crosnoe, C. Muller, E. Tipton, B. Schneider, C. Hulleman, C. Hinojosa, D. Paunesku, C. Romero, K. Flint, A. Roberts, J. Trott, R. Iachan, J. Buontempo, S. Yang, C. Carvalho, P. Hahn, M. Gopalan and P. Mhatre, "A national experiment reveals where a growth mindset improves achievement," Nature, vol. 108 , no. 3 , pp. 364-369, 2019. 